\title{
A Simple and Efficient Key Exchange Scheme Against the Smart Card Loss Problem
}

\author{
Ren-Chiun Wang ${ }^{1}$, Wen-Shenq Juang ${ }^{2}$, and Chin-Laung Lei ${ }^{1, \star}$ \\ ${ }^{1}$ Department of Electrical Engineering \\ National Taiwan University \\ No. 1, Sec. 4, Roosevelt Rd., Taipei, Taiwan 106, R.O.C. \\ rcwang@fractal.ee.ntu.edu.tw, lei@cc.ee.ntu.edu.tw \\ ${ }^{2}$ Department of Information Management \\ Shih Hsin University \\ No. 1, Lane17, Sec. 1, Mu-Cha Rd., Taipei, Taiwan 116, R.O.C. \\ wsjuang@cc.shu.edu.tw
}

\begin{abstract}
In a ubiquitous computing environment, a person can use various intelligent devices to obtain his desired services at any time and any place. For convenience, most of these devices are small and of limited power and computation capacity. Therefore, an admired scheme should take these into consideration. In 2006, Lin et al. proposed a lightweight authentication scheme only using one-way hash function. However, their scheme is vulnerable to the several security threats. It is the germination of our idea. In this paper, we only require one-way hash function, exclusive OR operation, a smart card, and a memorial password to construct a simple and efficient key exchange scheme to withstand the most known security threats. We also take several merits into our scheme. First, the friendliness and fairness of a user are considered. The user can freely select her/his identity and password for registration and employ the used identity to register repeatedly when the smart card has lost. Second, a user does not need to worry about the damage of the smart card loss problem even if the content of the smart card has been extracted. Our scheme can take care hard security threats and efficient at the same time. Since our scheme does not require any symmetric and asymmetric cryptosystems, the communication and computation cost is very low. Therefore, our scheme is suitable to be applied in ubiquitous computing environments.
\end{abstract}

Keywords: authentication, hash function, key exchange, password, smart card.

\section{Introduction}

In a ubiquitous computing environment, each user can use many mobile devices to obtain his service at any time and any place without knowing how to use these devices 18. These devices could have a low communication and computation capability. When a user wants to get a permitted service from a server,

\footnotetext{
^ Corresponding author.
} 
authentication and key exchange are basic mechanisms due to that the public networks are teem with many uncertainties and security threats are to come out one after the other. In the previous authenticated key exchange schemes, asymmetric cryptosystems such as the Diffie-Hellman [8], ElGamal [1], and RSA [27] schemes are often adopted. However, in those schemes [7,26], the computational complexity and the storage cost are burden.

For mulching the implementation easy and enhancing the performance, many authenticated key exchange schemes were proposed [1517] by employing symmetric cryptosystems such as DES [10 and AES 1, a memorial password, a one-way hash function 3] and a smart card [21. However, in those schemes, scholars always discuss to withstand most known security threats over the public networks such as the replay, the impersonation 1923, the dictionary 29, the known-key, and the stolen-verifier [20] attacks, and to enhance the performances of the schemes. Beside the above security threats, in a real life, a user always chooses the same identity and password and employs them to register with different application servers. Unfortunately, this user has to worry about whether the registered information (such as his password) are compromised or not and the security threats of the smart card is stolen by an attacker (also called the smart card loss problem). The administrator of a system could get the password of a registered user and impersonate this user to obtain the service from other servers [29. The smart card loss problem means that an adversary could employ the information of the smart card to launch some attacks such as the impersonation attack 3031. By the way, if a smart card is lost, the holder has to register with the server again using different identities appeared previous schemes. That is not convenient for a user. Therefore, revoking the loss card without changing the user's identity that should be an important issue to take it into consideration.

In 2005, Fan et al. 12 proposed a robust authentication scheme based on the concept of symmetric cryptosystem, quadratic residue [13], one-way hash function and exclusive OR operation. In their scheme, a solution was proposed to solve the smart card loss problem. However, the insider attack is still existed, and password changing and key exchange is not supported. Not only that, the storage, the computation and the communication costs of Fan et al.'s scheme are still burden. In 2006, Lin et al. 24 proposed a lightweight authentication scheme which is constructed by one-way hash function and simple exclusive OR operation without using any symmetric and asymmetric cryptosystems. In their scheme, a solution was proposed to prevent the insider attack. Unfortunately, we show that their scheme is vulnerable to the impersonation, the stolen-verifier, and the smart card lose problem.

From the above description, a secure and efficient smart card-based authenticated key exchange scheme should take the following properties into considerations:

$C_{1}$ : The communication and the computation costs are very low.

$C_{2}$ : Passwords can be chosen and changed freely by the users themselves.

$C_{3}$ : The serious time synchronization problem is not existed in the scheme. 
$C_{4}$ : The client and the server can confirm the owned session key is correct.

$C_{5}$ : The scheme can withstand the smart card loss problem.

$C_{6}$ : The user can revoke his loss card without changing the identity.

$C_{7}$ : The scheme can withstand the administrator of a system could get the password of a registered user.

$C_{8}$ : The scheme can withstand the dictionary attack without the smart card.

$C_{9}$ : The scheme can withstand the replay attack.

$C_{10}$ : The scheme can withstand the impersonation attack.

$C_{11}$ : The scheme can withstand the known-key attack.

$C_{12}$ : The scheme can withstand the stolen-verifier attack.

In this paper, we propose a simple and efficient authentication and key exchange scheme without using any symmetric or asymmetric cryptosystems. The proposed scheme provides all of the above properties. The communication and the computation cost is very low in our scheme. Therefore, the proposed scheme is suitable to be applied to ubiquitous computing environments.

The rest of this paper is organized as follows. In the next section, we introduce some definitions and theorems which are used in our scheme. In Section 3, we review Lin et al.'s scheme and show that their scheme is insecure. In Section 4, we describe our scheme. In Section 5, we analyze the security of our scheme. In Section 6, we evaluate the performances of our scheme. Finally, we conclude this paper in Section 7.

\section{Preliminaries}

In this section, we introduce some definitions and theorems of the exclusive OR operation, and one-way hash function in our scheme.

\subsection{Exclusive OR Operation}

We denote that $W$ is a result of $X$ bit-wise exclusive OR $Y$. In 2000, Ghanem and Wahab 14 have showed that the exclusive OR operation is secure and the computation is fast. The exclusive OR operation provides the following properties:

1. $W, X$, and $Y$ are the same bit length.

2. All output results are uniformly distributed in the output domain.

3. We can employ any two of $W, X$, and $Y$ to retrieve the other one, it is very easy.

4. If the length of $W$ is $n$ bits, there are $2^{n}$ different pairs to construct $W=X$ $\oplus Y$.

Theorem 1. Let $X$ and $Y$ are $n$ bits specific values and $W=X \oplus Y$. The probability is negligible to retrieve $X$ and $Y$ when $n$ is large and only given $W$.

Proof: According to the property 1. of the exclusive OR operation, when $X$ and $Y$ are $n$ bits, we can derive $W$ also is $n$ bits. In the property 4 . of the exclusive 
OR operation, there are $2^{n}$ possible pairs to construct $W=X \oplus Y$. There is a negligible probability which is $\frac{1}{2^{n}}$ to obtain the specific $X$ and $Y$ from the given $W$.

\subsection{Hash Function}

We denote that $\mathrm{h}()$ is a one-way hash function. The one-way hash function has the following properties:

1. The function $\mathrm{h}()$ can take a message of an arbitrary-length input and produce a message digest of a fixed-length output.

2. The function $\mathrm{h}()$ is one-way. Given $X$, it is easily to calculate $\mathrm{h}(X)=Y$. However, given $Y$, it is hard to derive $\mathrm{h}^{-1}(Y)=X$.

3. The function $\mathrm{h}()$, given $X$, it is computationally infeasible to find out $X^{\prime}$ which is not equal to $X$ to satisfy $\mathrm{h}\left(X^{\prime}\right)=\mathrm{h}(X)$.

4. The function $\mathrm{h}()$, it is computationally infeasible to find out any two pairs $X^{\prime} \neq X$ to satisfy $\mathrm{h}\left(X^{\prime}\right)=\mathrm{h}(X)$.

There are two well-known hash functions SHA-1 3] and Merkle's hash function 25. which are aimed high-speed software implementations and are current in the public domain. We know many cryptosystems employ hash function for achieving authentication. Now, we also apply it into our scheme.

\section{Review of Lin et al.'s Scheme}

In this section, we review Lin et al.'s scheme [23] and show that their scheme is vulnerable to the impersonation, the stolen verifier, and the smart card loss problem attacks.

\subsection{Lin et al.'s Scheme}

\section{Registration phase}

Step 1. A new user $U_{i}$ selects a password $P W_{i}$ and a nonce $N_{i}$ and calculates a verifier $\mathrm{h}\left(P W_{i} \| N_{i}\right)$ for registration, where $\mathrm{h}()$ is a one-way hash function and $\|$ denotes the concatenation of two strings. $U_{i}$ sends the verifier $\mathrm{h}\left(P W_{i} \| N_{i}\right)$ with his identity $I D_{i}$ to a server through a secure channel.

Step 2. The server stores the verifier $\mathrm{h}\left(P W_{i} \| N_{i}\right)$ into a database and calculates a secret value $K=\mathrm{h}\left(x \| I D_{i}\right) \oplus \mathrm{h}\left(P W_{i} \| N_{i}\right)$, where $x$ is the server's secret key. The server writes the $K$ into a personal smart card and issues it to $U_{i}$.

\section{Authentication phase}

When $U_{i}$ wants to get a service from the server, $U_{i}$ inserts his smart card and keys in his password $P W_{i}$. Then the smart card and the server can perform the following steps for authentication. 
Step 1. The smart card first retrieves the stored contents and selects a new nonce $N_{i}^{\prime}$. Then the smart card calculates $C_{1}=K \oplus \mathrm{h}\left(P W_{i} \| N_{i}\right)=$ $\mathrm{h}\left(x \| I D_{i}\right), C_{2}=\mathrm{h}(K) \oplus \mathrm{h}\left(P W_{i} \| N_{i}^{\prime}\right)=\mathrm{h}\left(\mathrm{h}\left(x \| I D_{i}\right) \oplus \mathrm{h}\left(P W_{i} \|\right.\right.$ $\left.\left.N_{i}\right)\right) \oplus \mathrm{h}\left(P W_{i} \| N_{i}^{\prime}\right)$, and $C_{3}=\mathrm{h}\left(C_{1} \oplus \mathrm{h}\left(P W_{i} \| N_{i}^{\prime}\right)\right)=\mathrm{h}\left(\mathrm{h}\left(x \| I D_{i}\right)\right.$ $\left.\oplus \mathrm{h}\left(P W_{i} \| N_{i}^{\prime}\right)\right)$. Finally, the smart card sends $\left(I D_{i}, C_{2}, C_{3}\right)$ to the server.

Step 2. After receiving the login request, the server retrieves $\mathrm{h}\left(P W_{i} \| N_{i}\right)$ from the database, and performs the following steps for verifying the identity of the $U_{i}$.

Step 2.1. Check the format of $I D_{i}$. If it is not true, the connection is terminated.

Step 2.2. Retrieve $\mathrm{h}\left(P W_{i} \| N_{i}^{\prime}\right)$ by computing $\mathrm{h}\left(\mathrm{h}\left(x \| I D_{i}\right) \oplus \mathrm{h}\left(P W_{i} \| N_{i}\right)\right) \oplus$ $\mathrm{C}_{2}$.

Step 2.3. Calculate $C_{3}^{\prime}=\mathrm{h}\left(\mathrm{h}\left(x \| I D_{i}\right) \oplus \mathrm{h}\left(P W_{i} \| N_{i}^{\prime}\right)\right)$ and verify whether $C_{3}^{\prime}$ is equal to $C_{3}$ or not. If it holds, the identity of $U_{i}$ is authenticated; otherwise, the login request is denied. Finally, the server updates the verifier $\mathrm{h}\left(P W_{i} \| N_{i}\right)$ with $\mathrm{h}\left(P W_{i} \| N_{i}^{\prime}\right)$.

\subsection{Security Analysis of Lin et al.'s Scheme}

We show that some security threats can work in the Lin et al.'s scheme as follows. We use $C_{x}^{j}$ to denote the $j$ th login information, where $x=2$ and 3 . The $i$ th login request should include $\left(I D_{i}, C_{2}^{j}, C_{3}^{j}\right)$.

\section{The impersonation attack}

Step 1. Assume that, an attacker could tap $(j$ th, $(j+1)$ th $)$ login information $\left(C_{2}^{j}=\mathrm{h}\left(\mathrm{h}\left(x \| I D_{i}\right) \oplus \mathrm{h}\left(P W_{i} \| N_{i}\right)\right) \oplus \mathrm{h}\left(P W_{i} \| N_{i}^{\prime}\right), C_{3}^{j}=\mathrm{h}(\mathrm{h}(x \|\right.$ $\left.\left.I D_{i}\right) \oplus \mathrm{h}\left(P W_{i} \oplus N_{i}^{\prime}\right)\right)$ and $\left(C_{2}^{j+1}=\mathrm{h}\left(\mathrm{h}\left(x \| I D_{i}\right) \oplus \mathrm{h}\left(P W_{i} \| N_{i}^{\prime}\right)\right) \oplus\right.$ $\mathrm{h}\left(P W_{i} \| N_{i}^{\prime \prime}\right), C_{3}^{j+1}=\mathrm{h}\left(\mathrm{h}\left(x \| I D_{i}\right) \oplus \mathrm{h}\left(P W_{i} \| N_{i}^{\prime \prime}\right)\right)$. Now, we can know the latest verifier is $\mathrm{h}\left(P W_{i} \| N_{i}^{\prime \prime}\right)$ which is used to verify $(j+2)$ th login request.

Step 2. The attacker could obtain the latest verifier $\mathrm{h}\left(P W_{i} \| N_{i}^{\prime \prime}\right)$ by computing $C_{3}^{j} \oplus C_{2}^{j+1}$.

Step 3. Now, the attacker could forge the $(j+2)$ th login information by calculating $C_{2}^{j+2}=C_{3}^{j+1} \oplus \mathrm{h}\left(P W_{i} \| N_{i}^{\prime \prime}\right)=\mathrm{h}\left(\mathrm{h}\left(x \| I D_{i}\right) \oplus \mathrm{h}\left(P W_{i} \| N_{i}^{\prime \prime}\right)\right)$ $\oplus \mathrm{h}\left(P W_{i} \| N_{i}^{\prime \prime}\right)$ and $C_{3}^{j+2}=C_{3}^{i+1}=\mathrm{h}\left(\mathrm{h}\left(x \| I D_{i}\right) \oplus \mathrm{h}\left(P W_{i} \| N_{i}^{\prime \prime}\right)\right)$. The attacker sends $\left(C_{2}^{j+2}, C_{3}^{j+2}\right)$ to the server.

Step 4. The server will first retrieve $\mathrm{h}\left(P W_{i} \| N_{i}^{\prime \prime}\right)$ from the database and compute $\mathrm{h}\left(\mathrm{h}\left(x \| I D_{i}\right) \oplus \mathrm{h}\left(P W_{i} \| N_{i}^{\prime \prime}\right)\right) \oplus C_{2}^{j+2}$ to get next verifier $\mathrm{h}\left(P W_{i}\right.$ $\left.\| N_{i}^{\prime \prime}\right)$. Then the server verifies whether $C_{3}^{j+2}$ is equal to $\mathrm{h}\left(\mathrm{h}\left(x \| I D_{i}\right)\right)$ $\oplus \mathrm{h}\left(P W_{i} \| N_{i}^{\prime \prime}\right)$ or not. If it holds, the identity of $U_{i}$ is authenticated; otherwise, the login request is denied. According to the forged $\left(C_{2}^{j+2}\right.$, $C_{3}^{j+2}$ ), we can know the server will accept this login request, and update the stored verifier $\mathrm{h}\left(P W_{i} \| N_{i}^{\prime \prime}\right)$ with $\mathrm{h}\left(P W_{i} \| N_{i}^{\prime \prime}\right)$.

Step 5. Using this way, the attacker can iteratively employ $C_{2}^{j+2}$ and $C_{3}^{j+2}$ for his later login requests without the smart card and the password of $U_{i}$. 


\section{The stolen-verifier attack}

Step 1. Assume that, the latest verifier is $\mathrm{h}\left(P W_{i} \| N_{i}^{\prime}\right)$ which is used to verify $(j+1)$ th login request.

Step 2. Now, the attacker has stolen the latest verifier $\mathrm{h}\left(P W_{i} \| N_{i}^{\prime}\right)$ and intercepts the last login information $\left(C_{2}^{j}, C_{3}^{j}\right)$.

Step 3. Then the attacker can forge the $(j+1)$ th login information $\left(C_{2}^{j+1}, C_{3}^{j+1}\right)$, where $C_{2}^{j+1}=C_{3}^{j} \oplus \mathrm{h}\left(P W_{i} \| N_{i}^{\prime}\right)=\mathrm{h}\left(\mathrm{h}\left(x \| I D_{i}\right) \oplus \mathrm{h}\left(P W_{i} \| N_{i}^{\prime}\right)\right) \oplus$ $\mathrm{h}\left(P W_{i} \| N_{i}^{\prime}\right)$ and $C_{3}^{j+1}=C_{3}^{j}=\mathrm{h}\left(\mathrm{h}\left(x \| I D_{i}\right) \oplus \mathrm{h}\left(P W_{i} \| N_{i}^{\prime}\right)\right)$.

Step 4. This attack is similar to the impersonation attack. As we know, the login request is accepted by the server and the attacker can iteratively employ $C_{2}^{j+1}$ and $C_{3}^{j+1}$ for his later login requests without the smart card and the password of $U_{i}$.

\section{The smart card loss problem}

Step 1. If the smart card is compromised by an attacker, the attacker can obtain the contents of the smart card, $K=\mathrm{h}\left(x \| I D_{i}\right) \oplus \mathrm{h}\left(P W_{i} \| N_{i}\right)$. The attacker also can intercepts the last and $j$ th login information $\left(C_{2}^{j}=\right.$ $\mathrm{h}(K) \oplus \mathrm{h}\left(P W_{i} \| N_{i}^{\prime}\right)=\mathrm{h}\left(\mathrm{h}\left(x \| I D_{i}\right) \oplus \mathrm{h}\left(P W_{i} \| N_{i}\right)\right) \oplus \mathrm{h}\left(P W_{i} \| N_{i}^{\prime}\right)$, $C_{3}^{j}=\mathrm{h}\left(\mathrm{h}\left(x \| I D_{i}\right) \oplus \mathrm{h}\left(P W_{i} \| N_{i}^{\prime}\right)\right)$. Now, we know the $(j+1)$ th verifier is $\mathrm{h}\left(P W_{i} \| N_{i}^{\prime}\right)$.

Step 2. The attacker can compute $\mathrm{h}(K) \oplus C_{2}^{i}$ to obtain the $(i+1)$ th verifier $\mathrm{h}\left(P W_{i} \| N_{i}^{\prime}\right)$.

Step 3. Then the attacker forges the $(j+1)$ th login information by calculating $C_{2}^{j+1}=C_{3}^{j} \oplus \mathrm{h}\left(P W_{i} \| N_{i}^{\prime}\right)=\mathrm{h}\left(\mathrm{h}\left(x \| I D_{i}\right) \oplus \mathrm{h}\left(P W_{i} \| N_{i}^{\prime}\right)\right) \oplus \mathrm{h}\left(P W_{i}\right.$ $\left.\| N_{i}^{\prime}\right)$ and $C_{3}^{j+1}=C_{3}^{j}=\mathrm{h}\left(\mathrm{h}\left(x \| I D_{i}\right) \oplus \mathrm{h}\left(P W_{i} \| N_{i}^{\prime}\right)\right)$.

Step 4. As we know, the forged login request will be accepted by the server and the attacker can iteratively employ $C_{2}^{i+1}$ and $C_{3}^{i+1}$ for his later login requests without the password of $U_{i}$.

\section{Our Proposed Scheme}

The intention of our scheme is to propose a simple and efficient key exchange scheme against the potential and serious threats that are the insider attack and the smart card loss problem. We divide the scheme into two phases: the registration phase and the authentication phase. We start to introduce the proposed scheme as follows.

\section{Registration phase}

Step 1. A new user $U_{i}$ selects a password $P W_{i}$ and a random number $N_{i}$ and calculates a verifier $\mathrm{h}\left(P W_{i} \| N_{i}\right)$ for registration, where $\mathrm{h}()$ is a one-way hash function and $\|$ denotes the concatenation of two strings. $U_{i}$ sends the verifier $\mathrm{h}\left(P W_{i} \| N_{i}\right)$ with his identity $I D_{i}$ to a server through a secure channel. 
Step 2. The server calculates a secret value $K=\mathrm{h}\left(x\left\|I D_{i}\right\| C I D_{i}\right)$, where $x$ is the server's secret key and $C I D_{i}$ is the smart card's identifier. The server writes $\left(I D_{i}, K\right)$ into a personal smart card and issues it to the $U_{i}$. The server stores $\left(I D_{i}, C I D_{i}, \mathrm{~h}\left(P W_{i} \| N_{i}\right)\right)$ into a database.

Step 3. $U_{i}$ writes $N_{i}$ into the smart card. Finally, the contents of the smart card is $\left(I D_{i}, K, N_{i}\right)$.

\section{Authentication phase}

When $U_{i}$ wants to establish a secure conversation with the server, $U_{i}$ inserts his smart card and keys in the password $P W_{i}$. Then the smart card and the server can perform the following steps for agreeing a common session key.

\section{Smart cards}

Step 1. Retrieve the contents $\left(I D_{i}, K, N_{i}\right)$ and select a random number $N_{i}^{\prime}$.

Step 2. Calculate $C_{1}=\mathrm{h}^{2}\left(P W_{i} \| N_{i}\right), C_{2}=C_{1} \oplus \mathrm{h}^{2}\left(P W_{i} \| N_{i}^{\prime}\right), K_{1}=$ $\mathrm{h}\left(\mathrm{h}\left(P W_{i} \| N_{i}\right) \| K\right)$, and $C_{3}=\mathrm{h}\left(K_{1} \| \mathrm{h}^{2}\left(P W_{i} \| N_{i}^{\prime}\right)\right)$.

Step 3. Send $\left(I D_{i}, C_{2}, C_{3}\right)$ to the server.

\section{Server}

Step 4. Check whether the $I D_{i}$ is existed in the database or not. If not, the connection is terminated; otherwise, retrieve $\left(I D_{i}, C I D_{i}, \mathrm{~h}\left(P W_{i} \| N_{i}\right)\right)$ from the database.

Step 5. Calculate $V_{1}, \mathrm{~h}\left(x\left\|I D_{i}\right\| C I D_{i}\right), K_{1}^{\prime}$, and $C_{3}^{\prime}$, where $V_{1}=\mathrm{h}^{2}\left(P W_{i} \|\right.$ $\left.N_{i}\right) \oplus C_{2}=\mathrm{h}^{2}\left(P W_{i} \| N_{i}^{\prime}\right), K_{1}^{\prime}=\mathrm{h}\left(\mathrm{h}\left(P W_{i} \| N_{i}\right) \| \mathrm{h}\left(x\left\|I D_{i}\right\| C I D_{i}\right)\right)$ and $C_{3}^{\prime}=\mathrm{h}\left(K_{1}^{\prime} \| V_{1}\right)$.

Step 6. Verify whether $C_{3}$ is the same as the $C_{3}^{\prime}$ or not. If not, the connection is terminated.

Step 7. Select a random number $N_{s}$ and calculate $\left(C_{4}, S K, S_{1}\right)$, where $C_{4}=N_{s}$ $\oplus V_{1}, S K=\mathrm{h}\left(K_{1}^{\prime}\left\|N_{s}\right\| V_{1}\right)$, and $S_{1}=\mathrm{h}\left(K_{1}^{\prime} \| S K\right)$.

Step 8. Send $\left(C_{4}, S_{1}\right)$ to $U_{i}$.

\section{Smart cards}

Step 9. Retrieve $N_{s}^{\prime}$ by computing $\mathrm{h}^{2}\left(P W_{i} \| N_{i}^{\prime}\right) \oplus C_{4}$.

Step 10. Calculate $S K=\mathrm{h}\left(K_{1}\left\|N_{s}^{\prime}\right\| \mathrm{h}^{2}\left(P W_{i} \| N_{i}^{\prime}\right)\right)$, and $T_{1}=\mathrm{h}\left(K_{1} \| S K\right)$.

Step 11. Verify whether $S_{1}$ is the same as the $T_{1}$ or not. If not, the connection is terminated.

Step 12. Calculate $C_{5}=\mathrm{h}\left(K_{1}\right) \oplus \mathrm{h}\left(P W_{i} \| N_{i}^{\prime}\right)$ and send $C_{5}$ back to the server. Step 13. Update $N_{i}$ with $N_{i}^{\prime}$.

\section{Server}

Step 14. Calculate $V_{2}=C_{5} \oplus \mathrm{h}\left(K_{1}^{\prime}\right)=\mathrm{h}\left(P W_{i} \| N_{i}^{\prime}\right)$.

Step 15. Verify whether $\mathrm{h}\left(V_{2}\right)$ is equal to $V_{1}$ or not. If not, the connection is terminated; otherwise, accept the session key $S K$ and update $\mathrm{h}\left(P W_{i}\right.$ \| $N_{i}$ ) with $V_{2}$. 


\section{Password changing phase}

When $U_{i}$ wants to renew his password, $U_{i}$ does not need to extra perform a password changing phase. $U_{i}$ can first choose a new password $P W_{i_{\text {new }}}$ and a new random number $N_{i}^{\prime}$. Then $U_{i}$ can perform the steps of the authentication phase to achieve the purpose of changing password. Finally, the server will store a new verifier $\mathrm{h}\left(P W_{i_{\text {new }}} \| N_{i}^{\prime}\right)$.

\section{$5 \quad$ Security Analysis}

We use the logic analysis method [4,?] to prove the authentication of the proposed scheme which is described in appendix A and the heuristic security analysis to show that our scheme can withstand most of the known security threats. Before we analyze the proposed scheme, we first assume that an adversary has an ability to collect all message flows between a client and a server. For instance, when the last message flow is intercepted, the adversary can obtain $\left(C_{2}\right.$ $=\mathrm{h}^{2}\left(P W_{i} \| N_{i}\right) \oplus \mathrm{h}^{2}\left(P W_{i} \| N_{i}^{\prime}\right), C_{3}=\mathrm{h}\left(K_{1} \| \mathrm{h}^{2}\left(P W_{i} \| N_{i}^{\prime}\right)\right), C_{4}=N_{s} \oplus$ $\left.\mathrm{h}^{2}\left(P W_{i} \| N_{i}^{\prime}\right), S_{1}=\mathrm{h}\left(K_{1}^{\prime} \| S K\right), C_{5}=\mathrm{h}\left(K_{1}\right) \oplus \mathrm{h}\left(P W_{i} \| N_{i}^{\prime}\right)\right)$, where $K=$ $\mathrm{h}\left(x\left\|I D_{i}\right\| C I D_{i}\right), K_{1}=\mathrm{h}\left(\mathrm{h}\left(P W_{i} \| N_{i}\right) \| K\right), S K=\mathrm{h}\left(K_{1}\left\|N_{s}^{\prime}\right\| \mathrm{h}^{2}\left(P W_{i} \|\right.\right.$ $\left.N_{i}^{\prime}\right)$ ), and $K_{1}=K_{1}^{\prime}$.

\subsection{Revoking the Loss Card Without Changing the User's Identity}

When a user registers from a server, the server will issue a personal smart card to him, where the content of the smart card is $\left(I D_{i}, K=\mathrm{h}\left(x\left\|I D_{i}\right\| C I D_{i}\right)\right.$, $\left.N_{i}\right)$, the $C I D_{i}$ is the smart card's identifier and the server stores $\left(I D_{i}, C I D_{i}\right.$, $\left.\mathrm{h}\left(P W_{i} \| N_{i}\right)\right)$ in his database.

When the smart card has lost, the user can use the same identity $I D_{i}$ to register again, the content of the new smart card becomes $\left(I D_{i}, K=\mathrm{h}\left(x \| I D_{i}\right.\right.$ $\left.\left.\| C I D_{i}^{\prime}\right), N_{i_{\text {new }}}\right)$, where the $C I D_{i}^{\prime}$ is a new identifier of the smart card and the server's verifier are to become $\left(I D_{i}, C I D_{i}^{\prime}, \mathrm{h}\left(P W_{i} \| N_{i_{\text {new }}}\right)\right)$. Only the new card can achieve in the scheme.

This property provides us a protection: even if the adversary has older loss card or user's password, the server can employ $C I D_{i}^{\prime}$ to discriminate them.

\subsection{The Dictionary Attack}

On-line dictionary attack: This is an unavoidable attack and it requires the server joins this attack. The server can detect the failed times and take appropriate login times to prevent this attack, where the failed times means if the authentication phase is not finished, the failed times plus one. All passwordbased schemes can withstand this attack.

Off-line dictionary attack: If the adversary intercepts the communicated messages, the adversary can directly make a dictionary attack at off-line. However, this attack will be failed due to the adversary has no $\left(N_{i}, N_{i}^{\prime}, \mathrm{h}\left(x\left\|I D_{i}\right\|\right.\right.$ $\left.\left.C I D_{i}\right)\right)$. 


\subsection{The Smart Card Loss Problem}

After $j$ th login request is accepted by the server, if the smart card of a holder is stolen by an adversary, the adversary still can not launch some attacks on our scheme. Note that, the adversary can obtain the smart card's contents that are $\left(I D_{i}, K=\mathrm{h}\left(x\left\|I D_{i}\right\| C I D_{i}\right), N_{i}^{\prime}\right)$ and the $(j+1)$ th verifier is $\mathrm{h}\left(P W_{i} \|\right.$ $N_{i}^{\prime}$ ) which is stored in the server. We analyze some possible situations as follows.

Situation 1: The adversary directly makes the dictionary attack on the intercepted information $\left(C_{2}, C_{3}, C_{4}, S_{1}, C_{5}\right)$ with the smart card. We can find this attack is not succeed due to the adversary does not have the random number $N_{i}$.

Situation 2: The adversary's target is to get one of $\mathrm{h}\left(P W_{i} \| N_{i}\right), P W_{i}$ and $N_{i}$ and employs it to verify the guessed password from the intercepted messages with the smart card. However, we observe the adversary can not derive some valuable information due to the properties of the one-way hash function.

Situation 3: If the adversary wants to forge a login request, the adversary may have to guess a password $P W_{i}^{\prime}$ and to select a random number $N_{i_{\text {attacker }}}$. Then the adversary computes $\left(C_{2_{\text {attacker }}}=\mathrm{h}^{2}\left(P W_{i}^{\prime} \| N_{i_{\text {attacker }}}\right) \oplus \mathrm{h}^{2}\left(P W_{i}^{\prime} \|\right.\right.$ $\left.N_{i}^{\prime}\right), C_{3_{\text {attacker }}}=\mathrm{h}\left(K_{1_{\text {attacker }}} \| \mathrm{h}^{2}\left(P W_{i}^{\prime} \| N_{i_{\text {attacker }}}\right)\right)$ and sends $\left(I D_{i}, C_{2_{\text {attacker }}}\right.$, $\left.C_{3_{\text {attacker }}}\right)$ to the server, where $K_{1_{\text {attacker }}}=\mathrm{h}\left(\mathrm{h}\left(P W_{i}^{\prime} \| N_{i_{\text {attacker }}}\right) \| K\right)$.

Before the server sends a response $\left(C_{4}, S_{1}\right)$ back, the server first verifies whether $C_{3}$ is equal to $C_{3}^{\prime}$ or not. Now, if the adversary receives $\left(C_{4}, S_{1}\right)$, it denotes the adversary guesses the password correctly. Obviously, this is a online password guessing attack. The server can detect the failed times and permit appropriate login times to prevent this attack.

\subsection{The Insider Attack}

When a valid client submits his identity $I D_{i}$ and a verifier $\mathrm{h}\left(P W_{i} \| N_{i}\right)$ to the server, the server's administrator can not get $P W_{i}$ or launch a off-line dictionary attack on $\mathrm{h}\left(P W_{i} \| N_{i}\right)$ without the random number $N_{i}$. Therefore, the insider attack can not work in our scheme.

\subsection{The Replay Attack}

After $j$ th login request is accepted by the server, we know the $(j+1)$ th verifier becomes $\mathrm{h}\left(P W_{i} \| N_{i}^{\prime}\right)$. Now, if the adversary replays $\left(I D_{i}, C_{2}, C_{3}\right)$ to the server, the server will calculate $\mathrm{h}^{2}\left(P W_{i} \| N_{i}^{\prime}\right) \oplus C_{2}$ to get $\mathrm{h}^{2}\left(P W_{i} \| N_{i}\right)$ and verify whether $C_{3}^{\prime}=\mathrm{h}\left(K_{1} \| \mathrm{h}^{2}\left(P W_{i} \| N_{i}\right)\right)$ is the same as $C_{3}$. Then we can find $C_{3}$ is not equal to $C_{3}^{\prime}$. The adversary can not replay a used login request to impersonate this client.

\subsection{The Impersonation Attack}

In our scheme, even if the adversary gets all message flows from the communicated channel with the smart card, the adversary still can not launch a off-line 
dictionary attack or derive some valuable information due to the properties of one-way hash function and the detail is described in 5.3.

\subsection{The Known-Key Attack}

Once a used session key $S K=\mathrm{h}\left(K_{1}\left\|N_{s}^{\prime}\right\| \mathrm{h}^{2}\left(P W_{i} \| N_{i}^{\prime}\right)\right)$ is compromised, the adversary still can not get any advantage. The adversary could employ the session key to launch the following attacks:

Situation 1: Make a off-line dictionary attack on the session key directly. The adversary can not succeed without the knowledge of $\left(K_{1}, N_{s}, N_{i}^{\prime}\right)$.

Situation 2: Retrieve some valuable information from the intercepted messages. The adversary can not succeed due to the properties of the one-way hash function.

\subsection{The Stolen-Verifier Attack}

When the latest verifier $\mathrm{h}\left(P W_{i} \| N_{i}^{\prime}\right)$ has stolen by an adversary, the adversary can perform the following situations to launch some attacks.

Situation 1: Make a off-line dictionary attack on the verifier. The adversary can not succeed without $N_{i}^{\prime}$.

Situation 2: Retrieve some valuable information from the intercepted messages by using the verifier. The adversary can get $\left(\mathrm{h}^{2}\left(P W_{i} \| N_{i}\right), N_{s}, \mathrm{~h}\left(K_{1}\right)\right)$ from $C_{2}$, $C_{4}$ and $C_{5}$ respectively. However, the adversary still can not launch the off-line dictionary attack from these information without $N_{i}$, derive the previous session keys and forge next login request without $K_{1}$.

\section{Performance Considerations}

In this section, we compare the computational complexity and satisfaction of the properties with the previous schemes for evaluating our scheme. We assume that: the output length of a one-way hash function is 160 bits; the output length of a symmetric cryptosystem is 128 bits; and the output length of an asymmetric cryptosystem is 1024 bits.

\subsection{Efficiency Comparison}

To analyze the computational complexity, we define some notations: $T_{h}$ denotes the time of one hashing function operation, $T_{\text {exp }}$ denotes the time of one modular exponential operation, $T_{\text {sym }}$ denotes the time of one symmetric encryption or decryption, $T_{s q r}$ denotes the time for one modular square root, $T_{\oplus}$ denotes the time of one XOR operation, The length of an identity is 32-bit, the length of a random number is 64-bit, and the length of a timestamp is 32-bit. Then we use table 1 to show that our comparison. 
Table 1. Comparisons of computation and communication costs between our scheme and the related schemes

\begin{tabular}{|c|c|c|c|}
\hline & $\begin{array}{l}\text { Registration } \\
\text { phase }\end{array}$ & $\begin{array}{l}\text { Authentication } \\
\text { phase }\end{array}$ & $\begin{array}{c}\text { The size of the } \\
\text { transferred messages }\end{array}$ \\
\hline Our scheme & $2 T_{h}$ & $17 T_{h}+6 T_{\oplus}$ & $\begin{array}{c}I D+5 \times 160 \\
=832 \mathrm{bits}\end{array}$ \\
\hline $\begin{array}{l}\text { Liaw et al.'s } \\
\text { scheme } 22\end{array}$ & $1 T_{h}+1 T_{\oplus}$ & $\begin{array}{c}3 T_{h}+2 T_{\oplus}+ \\
2 T_{\text {exp }}+4 T_{\text {symm }}\end{array}$ & $\begin{array}{c}I D+1 \times 160+R+128 \\
=384 \text { bits }\end{array}$ \\
\hline $\begin{array}{c}\text { Juang's } \\
\text { scheme [16] }\end{array}$ & $1 T_{h}+1 T_{\oplus}$ & $\begin{array}{c}5 T_{h}+1 T_{\oplus}+ \\
6 T_{\text {symm }}\end{array}$ & $\begin{aligned} I D & +R+3 \times 128 \\
& =480 \mathrm{bits}\end{aligned}$ \\
\hline $\begin{array}{l}\text { Chen-Yeh's } \\
\text { scheme [ } 6\end{array}$ & $1 T_{h}+2 T_{\oplus}$ & $13 T_{h}+6 T_{\oplus}$ & $\begin{array}{l}I D+4 \times 160 \\
=672 \mathrm{bits}\end{array}$ \\
\hline $\begin{array}{l}\text { Fan et al.'s } \\
\text { scheme [12] }\end{array}$ & $2 T_{h}+1 T_{\text {symm }}$ & \begin{tabular}{|}
$5 T_{h}+2 T_{\oplus}+1 T_{\text {sqr }}$ \\
$+1 T_{\text {mul }}+1 T_{\text {symm }}$
\end{tabular} & $\begin{array}{c}I D+3 \times 160+1 \times 1024 \\
=1536 \text { bits }\end{array}$ \\
\hline $\begin{array}{c}\text { Shieh-Wang's } \\
\text { scheme } 28\end{array}$ & $1 T_{h}+2 T_{\oplus}$ & $9 T_{h}+4 T_{\oplus}$ & $\begin{array}{c}I D+3 \times 160+4 \times T \\
=640 \mathrm{bits}\end{array}$ \\
\hline $\begin{array}{l}\text { Lin et al.'s* } \\
\text { scheme [24] }\end{array}$ & $2 T_{h}+1 T_{\oplus}$ & $7 T_{h}+6 T_{\oplus}$ & $\begin{array}{l}I D+2 \times 160 \\
=352 \mathrm{bits}\end{array}$ \\
\hline
\end{tabular}

* The scheme is only to provide an unilateral authentication.

Table 2. Comparisons of satisfaction of the properties between our scheme and the related schemes

\begin{tabular}{|c|c|c|c|c|c|c|c|c|c|c|c|c|}
\hline & $C_{1}$ & $C_{2}$ & $C_{3}$ & $C_{4}$ & $C_{5}$ & $C_{6}$ & $C_{7}$ & $C_{8}$ & $C_{9}$ & $C_{10}$ & $C_{11}$ & $C_{12}$ \\
\hline Our scheme & $\begin{array}{c}\text { Extremely } \\
\text { Low }\end{array}$ & Yes & Yes & Yes & Yes & Yes & Yes & Yes & Yes & Yes & Yes & Yes \\
\hline $\begin{array}{c}\text { Liaw et al.'s } \\
\text { scheme [2] }\end{array}$ & High & Yes & Yes & Yes & No & No & No & Yes & Yes & Yes & Yes & Yes \\
\hline $\begin{array}{c}\text { Juang's } \\
\text { scheme [16] }\end{array}$ & Low & No & Yes & Yes & No & No & No & Yes & Yes & Yes & Yes & Yes \\
\hline $\begin{array}{c}\text { Chen-Yeh's } \\
\text { scheme [6] }\end{array}$ & $\begin{array}{c}\text { Extremely } \\
\text { low }\end{array}$ & No & Yes & No & No & No & No & Yes & Yes & Yes & Yes & Yes \\
\hline $\begin{array}{c}\text { Fan et al.'s } \\
\text { scheme [12] }\end{array}$ & High & No & Yes & No & Yes & Yes & No & Yes & Yes & Yes & $*$ & Yes \\
\hline $\begin{array}{c}\text { Shieh-Wang's } \\
\text { scheme [28] }\end{array}$ & $\begin{array}{c}\text { Extremely } \\
\text { low }\end{array}$ & No & No & No & No & No & No & Yes & Yes & Yes & Yes & Yes \\
\hline $\begin{array}{c}\text { Lin } \text { et al.'s } \\
\text { scheme [24] }\end{array}$ & $\begin{array}{c}\text { Extremely } \\
\text { low }\end{array}$ & No & Yes & No & No & No & No & Yes & Yes & No & $*$ & No \\
\hline
\end{tabular}

* Denotes the scheme does not support a key exchange phase.

\subsection{Functionality Comparison}

A secure and efficient key exchange scheme should provide some properties which is described in the Section 1. Now, we compare satisfaction of the properties with the previous scholarship and use table 2 to show that our comparisons. 
From the Subsections 6.1 and 6.2, our scheme requires fewer computation cost to satisfy all of the properties. To do that, our scheme does not require any symmetric and asymmetric cryptosystems to protect the communicated messages. Therefore, our scheme is easy to be applied in ubiquitous computing environments.

\section{Conclusions}

In this paper, we have shown that Lin et al.'s scheme is insecure. We also have proposed a simple and efficient key exchange scheme to withstand the administrator of a system could get the password of a registered user and the smart card loss problem without using any symmetric and asymmetric cryptosystems. We also take the friendliness and fairness of the users into our consideration. Our scheme's the computation cost and the communicated message flows are low. Therefore, our scheme is suitable to be applied in many ubiquitous computing environments.

Acknowledgement. This work is supported in part by the National Science Council under the Grant NSC 96-2628-E-002-182-MY3, NSC 95-2221-E-128-004MY2, and by the Taiwan Information Security Center (TWISC), National Science Council under the Grants No. NSC 96-2219-E-001-001.

\section{References}

1. Advanced Encryption Standard, http://csrc.nist.gov/encryption/aes/

2. Bellare, M., Pointcheval, D., Rogaway, P.: Authenticated and key exchange secure against dictionary attacks. In: Preneel, B. (ed.) EUROCRYPT 2000. LNCS, vol. 1807, pp. 139-155. Springer, Heidelberg (2000)

3. Biham, E., Chen, R., Joux, A., Carribault, P., Jalby, W., Lemuet, C.: Collisions in SHA-0 and reduced SHA-1. In: Cramer, R.J.F. (ed.) EUROCRYPT 2005. LNCS, vol. 3494, pp. 36-57. Springer, Heidelberg (2005)

4. Burrows, M., Abadi, M., Needham, R.: A logic of authentication. ACM Transactions on Computer Systems (TOCS) 8(1), 18-36 (1990)

5. Buttyán, L., Staamann, S., Wilhelm, U.: A simple logic for authentication protocol design. In: Proc. of 11th IEEE Computer Security Foundations Workshop, Rockport, Massachusetts, USA, June 9-11, pp. 153-162 (1998)

6. Chen, Y.-C., Yeh, L.-Y.: An efficient nonce-based authentication with key agreement. Applied Mathematics and Computation 169(2), 982-994 (2005)

7. Chien, H.-Y., Wang, R.-C., Yang, C.-C.: Note on robust and simple authentication protocol. The Computer Journal 48(1), 27-29 (2005)

8. Diffie, W., Hellman, M.E.: New directions in cryptography. IEEE Trans. on Information Theory IT-22, 644-654 (1976)

9. Ding, Y., Horster, P.: Undetected on-line password guessing attacks. ACM Operating Systems Review 29(4), 77-86 (1995)

10. Eberle, H.: A high-speed DES implement for network applications. In: Brickell, E.F. (ed.) CRYPTO 1992. LNCS, vol. 740, pp. 527-545. Springer, Heidelberg (1993) 
11. ElGamal, T.: A public-key cryptosystem and a signature scheme based on discrete logarithms. IEEE Trans. on Information Theory IT-31, 469-472 (1985)

12. Fan, C.-I, Chan, Y.-C., Zhang, Z.-K.: Robust remote authentication scheme with smart cards. Computers \& Security 24, 619-628 (2005)

13. Fan, C.-I., Lei, C.-L.: Efficient blind signature schemes based on quadratic residues. IEE Electronics Letters 32(9), 811-813 (1996)

14. Ghanem, S.M., Wahah, H.A.: A simple XOR-based technique for distributing group key secure multicasting. In: Proc. of $5^{\text {th }}$ IEEE Symposium on Computers and Communications, pp. 166-171 (2000)

15. Juang, W.-S.: Efficient multi-server password authenticated key agreement using smart cards. IEEE Trans. on Consumer Electronics 50(1), 251-255 (2004)

16. Juang, W.-S.: Efficient password authenticated key agreement using smart cards. Computers \& Security 23, 167-173 (2004)

17. Juang, W.-S.: Efficient three-party key exchange using smart cards. IEEE Trans. on Consumer Electronics 50(2), 619-624 (2004)

18. Juang, W.-S.: Efficient User Authentication and Key Agreement in Ubiquitous Computing. In: Gavrilova, M., Gervasi, O., Kumar, V., Tan, C.J.K., Taniar, D., Laganà, A., Mun, Y., Choo, H. (eds.) ICCSA 2006. LNCS, vol. 3983, pp. 396-405. Springer, Heidelberg (2006)

19. Ku, W.-C., Chang, S.-T.: Impersonation attack on a dynamic ID-based remote user authentication scheme using smart cards. IEICE Trans. on communications E88$\mathrm{B}(5), 2165-2167$ (2005)

20. Ku, W.-C., Tsai, H.-C., Tsaur, M.-J.: Stolen-verifier attack on an efficient smart card-based one-time password authentication scheme. IEICE Trans. on communications E87-B(8), 2374-2376 (2004)

21. Lamport, L.: Password authentication with insecure communication. Communications of the ACM 24, 770-772 (1981)

22. Liaw, H.-T., Lin, J.-F., Wu, W.-C.: An efficient and complete remote user authentication scheme using smart cards. Mathematical and Computer Modelling 44, 223-228 (2006)

23. Lin, C.-L., Hung, C.-P.: Impersonation attack on two-gene-relation password authentication protocol 2GR. IEICE Trans. on communications E89-B(12), 34253427 (2006)

24. Lin, C.-W., Tsai, C.-S., Hwang, M.-S.: A new strong-password authenticaton scheme using one-way hash functions. International Journal of Computer and Systems Sciences 45(4), 623-626 (2006)

25. Merkle, R.C.: One-way hash functions and DES. In: Brassard, G. (ed.) CRYPTO 1989. LNCS, vol. 435, pp. 428-446. Springer, Heidelberg (1990)

26. Peyravian, M., Jeffries, C.: Secure remote user access over insecure networks. Computers Communications 29, 660-667 (2006)

27. Rivest, R.L., Shamir, A., Adleman, L.: A method for obtaining digital signature and public key cryptosystems. Communications of the ACM 21, 120-126 (1978)

28. Shieh, W.-G., Wang, J.-M.: Efficient remote mutual authentication and key agreement. Computers \& Security 25, 72-77 (2006)

29. Ku, W.-C., Chen, C.-M., Lee, H.-L.: Cryptanalysis of a variant of Peyravian-Zunic's password authentication scheme. IEICE Trans. on Communications E86-B, 1682 1684 (2003)

30. Ku, W.-C., Chen, S.-M.: Weakness and improvement of an efficient password based remote user authentication scheme using smart cards. IEEE Trans. on Consumer Electronics 50(1), 204-207 (2004) 
31. Messerges, T.S., Dabbish, E.A., Sloan, R.H.: Examining smart card security under the threat of power analysis attacks. IEEE Trans. on Computers 51(5), 541-552 (2002)

\section{Appendix A}

We use the logic analysis method [4.5] to prove the authentication of the proposed scheme.

\section{Notations}

- $P, Q$ : two principals.

- $C$ : channel.

- $C_{c}$ : a channel $C$ which can be read only if $c$ is known.

- $X$ : message, which could be data or formula or both.

- $C(X)$ or $C_{C}(X)$ : message $X$ on channels $C$ or $C_{C}$.

- $r(C)$ and $w(C)$ : the set of readers and writers of channel $C$.

- $P \in r(C)$ : a principal $P$ is one of readers of channel $C$.

- $P \in w(C)$ : a principal $P$ is one of writers of channel $C$.

- $\phi$ : formula.

- $P \triangleleft C(X): P$ sees $C(X)$.

- $P \triangleleft X \mid C: P$ sees $X$ through $C$.

- $P \triangleleft X: P$ sees $X$.

- $\sharp(X): X$ is fresh.

- $P \sim X: P$ once said $X$.

- $P \| \sim X: P$ has recently said $X$.

- $P \equiv \phi: P$ believes $\phi$.

- $\phi_{1} \longrightarrow \phi_{2}: \phi_{1}$ implies $\phi_{2}$.

We describe the synthetic rules which are used to prove the proposed scheme as follows:

- (Syn. 1): In order to achieve a goal $G$, all new goals $G_{1}, G_{2}, \ldots$ have to be achieved.

G

$\hookrightarrow G_{1}$

$\hookrightarrow G_{2}$

$\hookrightarrow \ldots$

- (Syn. 2:) If a principal $P$ wants to see a message $X$ arrived through a channel $C, P$ has to see $C(X)$ and can read $C$.

$P \models(P \triangleleft X \mid C)$

$$
\begin{aligned}
& \hookrightarrow P \triangleleft C(X) \\
& \hookrightarrow P \in r(C)
\end{aligned}
$$

- (Syn. 3:) If a principal $P$ wants to believe a formula $\phi$, the $P$ has to believe a formula $\phi^{\prime}$ and the implication $\phi^{\prime} \longrightarrow \phi$.

$P \models \phi$

$$
\begin{aligned}
& \hookrightarrow P \models \phi^{\prime} \\
& \hookrightarrow P \models\left(\phi^{\prime} \longrightarrow \phi\right)
\end{aligned}
$$


Proof of the authentication phase: When a user $U_{i}$ wants to access the resource from a remote server $S$, the transmitted messages between them are as follows:

(i) $U_{i} \longrightarrow S: I D_{i}, C_{1} \oplus \mathrm{h}^{2}\left(P W_{i} \| N_{i}^{\prime}\right), C_{3}$

(ii) $S \longrightarrow U_{i}: N_{s} \oplus V_{1}, \mathrm{~h}\left(K_{1}^{\prime} \| S K\right)$

(iii) $U_{i} \longrightarrow S: \mathrm{h}\left(K_{1}\right) \oplus \mathrm{h}\left(P W_{i} \| N_{i}^{\prime}\right)$

We transfer the messages to the description in the following.

(i) $S \triangleleft C_{C_{1}}\left(\mathrm{~h}^{2}\left(P W_{i} \| N_{i}^{\prime}\right)\right)$

(ii) $U_{i} \triangleleft C_{V_{1}}\left(N_{s}\right), C_{K_{1}^{\prime}}(S K)$

(iii) $S \triangleleft C_{K_{1}}\left(\mathrm{~h}\left(P W_{i} \| N_{i}^{\prime}\right)\right)$

We also identify the following assumptions.

(A1) $U_{i} \in r\left(C_{C_{1}}\right): U_{i}$ can read channel $C_{C_{1}}$.

(A2) $S \in r\left(C_{C_{1}}\right): S$ can read channel $C_{C_{1}}$.

(A3) $U_{i} \equiv\left(w\left(C_{C_{1}}\right)\right)=\left(U_{i}, S\right): U_{i}$ believes that only $S$ and $U_{i}$ can write channel $C_{C_{1}}$.

(A4) $S \models\left(w\left(C_{C_{1}}\right)\right)=\left(U_{i}, S\right): S$ believes that only $U_{i}$ and $S$ can write channel $C_{C_{1}}$.

(A5) $U_{i} \in r\left(C_{V_{1}}\right): U_{i}$ can read channel $C_{V_{1}}$.

(A6) $S \in r\left(C_{V_{1}}\right): S$ can read channel $C_{V_{1}}$.

(A7) $U_{i} \models\left(w\left(C_{V_{1}}\right)\right)=\left(U_{i}, S\right): U_{i}$ believes that only $S$ and $U_{i}$ can write channel $C_{V_{1}}$.

(A8) $S \models\left(w\left(C_{V_{1}}\right)\right)=\left(U_{i}, S\right): S$ believes that only $U_{i}$ and $S$ can write channel $C_{V_{1}}$.

(A9) $U_{i} \in r\left(C_{K_{1}^{\prime}}\right): U_{i}$ can read channel $C_{K_{1}^{\prime}}$.

(A10) $S \in r\left(C_{K_{1}}\right): S$ can read channel $C_{K_{1}}$.

(A11) $U_{i} \mid \equiv\left(w\left(C_{K_{1}^{\prime}}\right)\right)=\left(U_{i}, S\right): U_{i}$ believes that only $S$ and $U_{i}$ can write channel $C_{K_{1}}$.

(A12) $S \equiv\left(w\left(C_{K_{1}}\right)\right)=\left(U_{i}, S\right): S$ believes that only $U_{i}$ and $S$ can write channel $C_{K_{1}^{\prime}}$.

(A13) $U_{i} \equiv\left(\left(U_{i} \triangleleft N_{s} \mid C_{V_{1}}\right) \longrightarrow U_{i} \stackrel{S K}{\longleftrightarrow} S\right): U_{i}$ believes that he sees $N_{s}$ through channel $C_{V_{1}}$ implies that $U_{i}$ and $S$ share $S K$.

$(A 14) S \equiv\left(\left(S \triangleleft \mathrm{h}^{2}\left(P W_{i} \| N_{i}^{\prime}\right) \mid C_{C_{1}}\right) \longrightarrow U_{i} \stackrel{S K}{\longleftrightarrow} S\right): S$ believes that he sees $\mathrm{h}^{2}\left(P W_{i} \| N_{i}^{\prime}\right)$ through channel $C_{C_{1}}$ implies that $U_{i}$ and $S$ share $S K$.

(A15) $U_{i} \equiv\left(\left(U_{i} \triangleleft S K \mid C_{K_{1}^{\prime}}\right) \longrightarrow\left(S \models U_{i} \stackrel{S K}{\longleftrightarrow} S\right)\right)$ : $U_{i}$ believes that he sees $S K$ through channel $C_{K_{1}^{\prime}}$ implies that $S$ believes that $U_{i}$ and $S$ share $S K$.

$(A 16) S \equiv\left(\left(S \triangleleft \mathrm{h}\left(P W_{i} \| N_{i}^{\prime}\right) \mid C_{K_{1}}\right) \longrightarrow\left(U_{i} \equiv U_{i} \stackrel{S K}{\longleftrightarrow} S\right)\right): S$ believes that he sees $\mathrm{h}\left(P W_{i} \| N_{i}^{\prime}\right)$ through channel $C_{K_{1}}$ implies that $U_{i}$ believes that $U_{i}$ and $S$ share $S K$.

There are four goals need to be achieved in our scheme for proving the authentication property. 
(Goal 1:) $U_{i} \models U_{i} \stackrel{S K}{\longleftrightarrow} S$;

(Goal 2:) $S \models U_{i} \stackrel{S K}{\longleftrightarrow} S$;

(Goal 3:) $U_{i} \equiv\left(S \models U_{i} \stackrel{S K}{\longleftrightarrow} S\right)$

(Goal 4:) $S \models\left(U_{i} \equiv U_{i} \stackrel{S K}{\longleftrightarrow} S\right)$

We use the above synthetic rules and assumptions to prove our goals.

1. Prove Goal 1: $U_{i} \models U_{i} \stackrel{S K}{\longleftrightarrow} S$

Proof. By using (Syn. 3), we know that:

$U_{i} \models U_{i} \stackrel{S K}{\longleftrightarrow} S$

$$
\begin{aligned}
& \hookrightarrow U_{i} \equiv\left(U_{i} \triangleleft N_{s} \mid C_{V_{1}}\right) \\
& \hookrightarrow U_{i} \equiv\left(\left(U_{i} \triangleleft N_{s} \mid C_{V_{1}}\right) \longrightarrow U_{i} \stackrel{S K}{\longleftrightarrow} S\right)
\end{aligned}
$$

We use the assumption $(A 13)$ to achieve the new goal (2). Then we employ (Syn. 2) to show that our new goal (1).

$U_{i} \models\left(U_{i} \triangleleft N_{s} \mid C_{V_{1}}\right)$

$$
\begin{aligned}
& \hookrightarrow U_{i} \triangleleft C_{V_{1}}\left(N_{s}\right) \\
& \hookrightarrow U_{i} \in r\left(C_{V_{1}}\right)
\end{aligned}
$$

The new goals (3) and (4) are existed and achieved in the message 2 of the scheme and the assumption $(A 5)$.

From the above, the Goal 1: $U_{i} \models U_{i} \stackrel{S K}{\longleftrightarrow} S$ is proved.

2. Prove Goal 2: $S \models U_{i} \stackrel{S K}{\longleftrightarrow} S$

Proof. By using (Syn. 3), we know that:

$S \models U_{i} \stackrel{S K}{\longleftrightarrow} S$

$$
\begin{aligned}
\hookrightarrow S & \equiv\left(S \triangleleft \mathrm{h}^{2}\left(P W_{i} \| N_{i}^{\prime}\right) \mid C_{C_{1}}\right) \\
& \hookrightarrow S \equiv\left(\left(S \triangleleft \mathrm{h}^{2}\left(P W_{i} \| N_{i}^{\prime}\right) \mid C_{C_{1}}\right) \longrightarrow U_{i} \stackrel{S K}{\longleftrightarrow} S\right)
\end{aligned}
$$

We use the assumption ( $A 14)$ to achieve the new goal (6). Then we employ (Syn. 2) to show that our new goal (5). $S \equiv\left(S \triangleleft \mathrm{h}^{2}\left(P W_{i} \| N_{i}^{\prime}\right) \mid C_{C_{1}}\right)$

$$
\begin{aligned}
& \hookrightarrow S \triangleleft C_{C_{1}}\left(\mathrm{~h}^{2}\left(P W_{i} \| N_{i}^{\prime}\right)\right) \\
& \hookrightarrow S \in r\left(C_{C_{1}}\right)
\end{aligned}
$$

The new goals (7) and (8) are existed and achieved in the message 1 of the scheme and the assumption (A2).

From the above, the Goal 2: $S \models U_{i} \stackrel{S K}{\longleftrightarrow} S$ is proved. 
3. Prove goal 3: $U_{i} \models\left(S \models U_{i} \stackrel{S K}{\longleftrightarrow} S\right)$

Proof. By using (Syn. 3), we know that:

$U_{i} \equiv\left(S \models U_{i} \stackrel{S K}{\longleftrightarrow} S\right)$

$$
\begin{gathered}
\hookrightarrow U_{i} \models\left(U_{i} \triangleleft S K \mid C_{K_{1}^{\prime}}\right) \\
\hookrightarrow U_{i} \equiv\left(\left(U_{i} \triangleleft S K \mid C_{K_{1}^{\prime}}\right)\right. \\
\left.\hookrightarrow S \models U_{i} \stackrel{S K}{\longleftrightarrow} S\right)
\end{gathered}
$$

We use the assumption ( $A 15)$ to achieve the new goal (10). Then we employ (Syn. 2) to show that our new goal (9).

$U_{i} \equiv\left(U_{i} \triangleleft S K \mid C_{K_{1}^{\prime}}\right)$

$$
\begin{aligned}
& \hookrightarrow U_{i} \triangleleft C_{K_{1}^{\prime}}(S K) \\
& \hookrightarrow U_{i} \in r\left(C_{K_{1}^{\prime}}\right)
\end{aligned}
$$

The new goals (11) and (12) are existed and achieved in the message 2 of the scheme and the assumption $(A 9)$.

From the above, the Goal 3: $U_{i} \equiv\left(S \models U_{i} \stackrel{S K}{\longleftrightarrow} S\right)$ is proved.

4. Prove goal 4: $S \models\left(U_{i} \models U_{i} \stackrel{S K}{\longleftrightarrow} S\right)$

Proof. By using (Syn. 3), we know that:

$S \models\left(U_{i} \models U_{i} \stackrel{S K}{\longleftrightarrow} S\right)$

$$
\begin{aligned}
\hookrightarrow S & \equiv\left(S \triangleleft \mathrm{h}\left(P W_{i} \| N_{i}^{\prime}\right) \mid C_{K_{1}}\right) \\
\hookrightarrow & S \equiv\left(S \triangleleft \mathrm{h}\left(P W_{i} \| N_{i}^{\prime}\right) \mid C_{K_{1}}\right) \\
\hookrightarrow & \left.U_{i} \equiv U_{i} \stackrel{S K}{\longleftrightarrow} S\right)
\end{aligned}
$$

We use the assumption (A16) to achieve the new goal (14). Then we employ (Syn. 2) to show that our new goal (13). $S \models\left(S \triangleleft \mathrm{h}\left(P W_{i} \| N_{i}^{\prime}\right) \mid C_{K_{1}}\right)$

$$
\begin{aligned}
& \hookrightarrow S \triangleleft C_{K_{1}}\left(\mathrm{~h}\left(P W_{i} \| N_{i}^{\prime}\right)\right) \\
& \hookrightarrow S \in r\left(C_{K_{1}}\right)
\end{aligned}
$$

The new goals (15) and (16) are existed and achieved in the message 3 of the scheme and the assumption $(A 10)$.

From the above, the Goal $4: S \models\left(U_{i} \models U_{i} \stackrel{S K}{\longleftrightarrow} S\right)$ is proved. 